\title{
ANTIMALARIAL ACTIVITY OF TRITERPENOIDS WITH THE CUCURBITANE SKELETON
}

\section{Ramalhete $C^{1}$, Lopes $D^{2}$, Mulhovo $S^{3}$, Rosário $V^{2}$ and Ferreira MJU'}

${ }^{1}$ iMed.UL, Faculdade de Farmácia, Universidade de Lisboa, Av. Prof. Gama Pinto, 1649-003, Lisboa, Portugal; ${ }^{2}$ UEI Malaria, CMDT.LA, Institute of Hygiene and Tropical Medicine, UNL, R. da Junqueira 96, 1349-008 Lisbon, Portugal. ${ }^{3}$ Escola Superior Técnica, Departamento de Ciências Agro-Pecuárias, Universidade Pedagógica, Av. de Moçambique, Mozambique.

\section{Introduction}

Malaria, caused by protozoan parasites of the genus Plasmodium, is a devastating infectious disease in developing countries. One of the biggest problems that have hindered the control of malaria is the emergence and spread of drug resistant Plasmodium strains, particularly Plasmodium falciparum. In order to overcome this problem, new therapeutic agents based on new mechanisms of action or with new structures are urgently needed. Plants have been an important source of medicines against malaria. Quinine and artemisinin, two of the most important antimalarials currently in use, were derived from plants [1,2].

Momordica balsamina L. (Cucurbitaceae), a vegetable used as food, has also been widely used in traditional medicine, mainly for the treatment of fever and malaria in Mozambique and South Africa [3].

\section{Results and Discussion}

Bioassay-guided fractionation of the methanol extract of Momordica balsamina led to the isolation of three new cucurbitane-type triterpenoids, balsaminols C-E (1-3). Their structures were elucidated on the basis of spectroscopic methods including 2D NMR experiments (COSY, HMQC, HMBC and NOESY), (Figs. 3-5).

Balsaminols $C-E$, together with ten cucurbitacins isolated from the same plant (4-13), were evaluated for their antimalarial activity against the Plasmodium falciparum chloroquine-sensitive strain 3D7 and the chloroquine-resistant clone Dd2.

As it can be observed in Table 1, most of the compounds displayed antimalarial activity. Compounds $\mathbf{9}$ and $\mathbf{1 2}$ revealed the highest antiplasmodial effects against both strains ( $\mathrm{IC}_{50}$ values: 4.6 , and $7.4 \mu \mathrm{M}, 3 \mathrm{D} 7$, respectively; 4.0 , and $8.2 \mu \mathrm{M}, \mathrm{Dd} 2$, respectively).

When comparing the results obtained for compounds $1-13$, rings $A$ and $B$ seem to play an important role in the antiplasmodial activity.

Furthermore, the preliminary toxicity toward human cells of compounds 1-13 was investigated in breast cancer cell line (MCF-7). Compounds were inactive or showed weak toxicity $\left(I_{50}\right.$ values $\left.>19.0\right)$.

We can conclude that the isolated compounds were responsible for the activity found in the extracts in a preliminary screening and may be interesting as leads for the

development of new antimalarials. Our study also supports the use of Momordica balsamina against malaria in traditional medicine

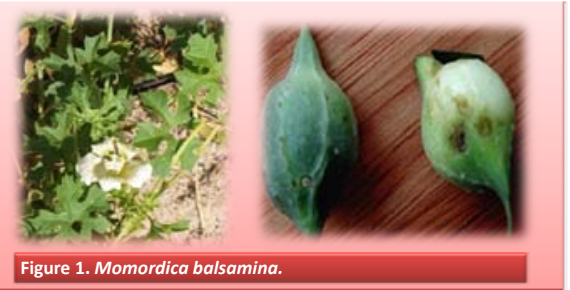

\section{Aim of the study}

Continuing our search for biologically active compounds from Momordica balsamina [4,5], the aim of this work was to find out antimalarial compounds from this medicinal African plant (Fig. 1).

Table 1. Antimalarial activity, cytotoxicity, and selectivity Index of compounds 1-13.

\begin{tabular}{|c|c|c|c|c|c|c|c|}
\hline \multirow{3}{*}{ Compound } & \multicolumn{5}{|c|}{$I C_{S 0} \pm S D$} & \multicolumn{2}{|c|}{ Selectivity Index } \\
\hline & \multicolumn{2}{|c|}{ P. falciparum 3D7 } & \multicolumn{2}{|c|}{ P. falciparum Dd2 } & \multirow{2}{*}{$\begin{array}{c}\text { MCF7 cells } \\
\mu \mathrm{M}\end{array}$} & \multirow[t]{2}{*}{ MCF $7 / 3 D 7$} & \multirow[t]{2}{*}{ MCF7/Dd2 } \\
\hline & $\mu \mathrm{M}$ & $\mu \mathrm{g} / \mathrm{mL}$ & $\mu \mathrm{M}$ & $\mu \mathrm{g} / \mathrm{mL}$ & & & \\
\hline 1 & $19.6 \pm 1.5$ & $9.2 \pm 0.7$ & $22.4 \pm 4.0$ & $10.5 \pm 1.9$ & $70.5 \pm 2.9$ & 3.6 & 3.2 \\
\hline 2 & $25.9 \pm 5.6$ & $11.1 \pm 2.4$ & $45.6 \pm 1.9$ & $19.6 \pm 0.8$ & $>133.3$ & $>5.1$ & $>2.9$ \\
\hline 3 & $20.4 \pm 2.1$ & $9.3 \pm 1.0$ & $19.6 \pm 4.6$ & $9.0 \pm 2.1$ & $44.2 \pm 3.9$ & 2.2 & 2.3 \\
\hline 4 & $19.1 \pm 3.4$ & $9.0 \pm 1.6$ & $27.3 \pm 6.1$ & $12.9 \pm 2.9$ & $65.1 \pm 1.6$ & 3.4 & 2.4 \\
\hline 5 & $18.7 \pm 1.6$ & $9.1 \pm 0.8$ & $19.2 \pm 2.7$ & $9.4 \pm 1.3$ & $34.0 \pm 0.1$ & 1.8 & 1.8 \\
\hline 6 & $14.6 \pm 1.3$ & $7.2 \pm 0.6$ & $33.0 \pm 6.3$ & $16.2 \pm 3.1$ & $47.0 \pm 0.4$ & 3.2 & 1.4 \\
\hline 7 & $17.1 \pm 0.4$ & $8.1 \pm 0.2$ & $23.5 \pm 4.9$ & $11.4 \pm 2.4$ & $40.0 \pm 1.3$ & 2.3 & 1.7 \\
\hline 8 & $15.2 \pm 5.4$ & $7.4 \pm 2.6$ & $17.9 \pm 3.7$ & $8.7 \pm 1.8$ & $31.0 \pm 3.7$ & 2.0 & 1.7 \\
\hline 9 & $4.6 \pm 0.5$ & $2.9 \pm 0.3$ & $4.0 \pm 0.5$ & $2.5 \pm 0.3$ & $19.0 \pm 0.8$ & 4.2 & 4.7 \\
\hline 10 & $13.2 \pm 0.8$ & $6.3 \pm 0.4$ & $17.6 \pm 0.7$ & $8.3 \pm 0.3$ & $>133.3$ & $>10.1$ & $>7.6$ \\
\hline 11 & $17.7 \pm 1.3$ & $8.6 \pm 0.6$ & $28.3 \pm 4.6$ & $13.8 \pm 2.2$ & $55.4 \pm 2.9$ & 3.1 & 2.0 \\
\hline 12 & $7.4 \pm 0.8$ & $3.3 \pm 0.4$ & $8.2 \pm 0.7$ & $3.8 \pm 0.3$ & $30.7 \pm 2.3$ & 4.2 & 3.7 \\
\hline 13 & $30.6 \pm 6.4$ & $14.0 \pm 2.4$ & $50.1 \pm 0.1$ & $23.0 \pm 0.04$ & $45.3 \pm 0.7$ & 1.5 & 0.9 \\
\hline CQ & $16 \mathrm{nM}$ & - & $200 \mathrm{nM}$ & - & - & - & - \\
\hline
\end{tabular}

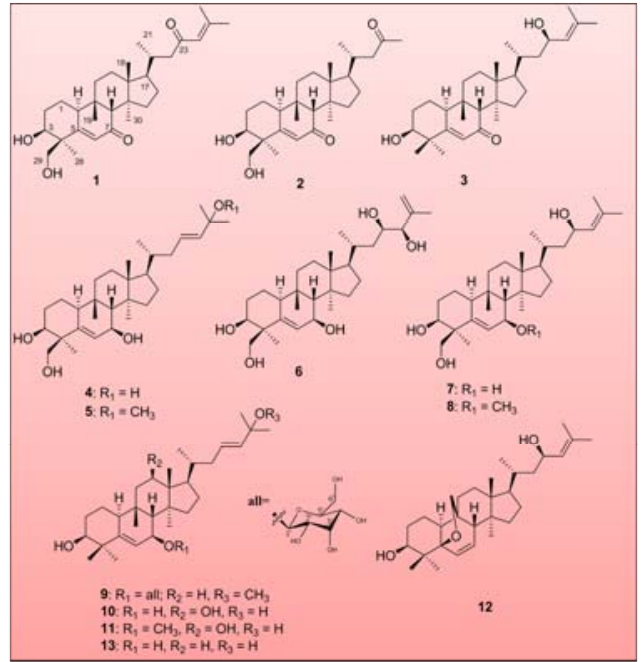

Figure 2. Structure of compounds isolated from M. balsamina 1-13

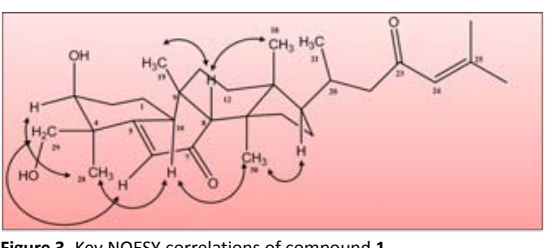

Figure 3. Key NOESY correlations of compound 1.

\section{Experimental Procedures}

Isolation of Compounds: The powdered aerial parts of Momordica balsamina were extracted at room temperature with methanol. The methanol extract was further fractionated by liquid-liquid extraction and chromatographic methods, until the isolation of pure compounds (Fig. 2). All the structures were deduced from their physical and spectroscopic data.

Antimalarial Assay: The evaluation of the antimalarial activity of compounds was performed against chloroquine-sensitive (3D7) and resistant (Dd2) P. falciparum strains by using a standardized SYBR Green I-based fluorescence assay [6].

In vitro Cytotoxicity Assay: Compounds were evaluated for their in vitro cytotoxic activity against human breast cancer cells (MCF-7) by using the MTT assay [6]

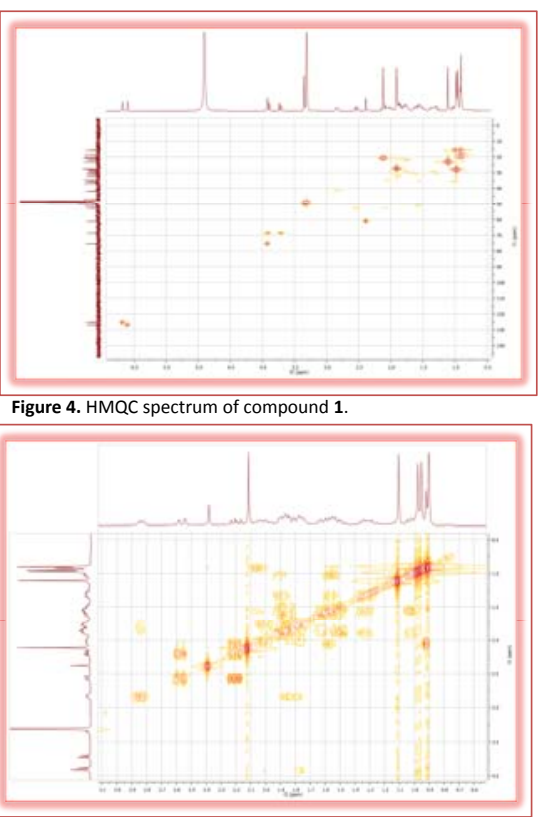

